\title{
Favorable effects of close telephone follow-up on Helicobacter pylori eradication success
}

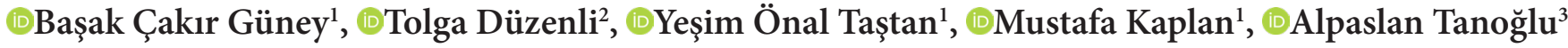 \\ ${ }^{1}$ Sultan Abdulhamid Han Training and Research Hospital, Department of Internal Medicine, Istanbul, Turkey \\ ${ }^{2}$ Hitit University Erol Olcok Training and Research Hospital, Department of Gastroenterology, Çorum, Turkey \\ ${ }^{3}$ Sultan Abdulhamid Han Training and Research Hospital, Department of Gastroenterology, Istanbul, Turkey
}

Cite this article as: Çakır Güney B, Düzenli T, Önal Taştan Y, Kaplan M, Tanoğlu A. Favorable effects of close telephone follow-up on Helicobacter pylori eradication success. Anatolian Curr Med J 2021; 3(1); 10-14.

\begin{abstract}
Introduction: To determine whether close follow-up by telephone calls is of benefit in Helicobacter pylori (HP) eradication rates.

Material and Method: This is a prospective, randomized, controlled clinical trial. Patients were randomized into two groups as patients who were followed up by telephone calls (TFG) and those who were not (NTFG; controls). Patients in the TFG group were called every 3 days for the 14 days during Hp treatment and were supported for treatment. Patients in the NTFG group were explained the treatment protocol in detail at treatment initiation and were instructed to return for a follow-up visit 4 weeks after treatment end. The latter group was not given support via telephone calls. All patients were examined by fecal HP antigen assay 4 weeks after eradication treatment.

Results: The 242 patients' age range was $19-82$ and their mean age was $45.01 \pm 14.6$ years. Of the patients, $52.1 \%(n=126)$ were women and $47.9 \%(n=116)$ were men. At treatment initiation and during medical examinations, $6.2 \%(n=15)$ of the patients voluntarily withdrew from the study. Treatment was discontinued in $5.8 \%(n=14)$ during the course of treatment due to side effects. Of the remaining 213 patients, 108 were randomized to the TFG group and 105 to the NTFG group. Eradication was achieved in $80 \%(\mathrm{n}=84)$ and could not be achieved in $20 \%(\mathrm{n}=21)$ of the patients in NTFG. Eradication was achieved in $91.6 \%$ $(\mathrm{n}=99)$ and could not be achieved in $8.4 \%(\mathrm{n}=9)$ of the patients in TGF $(\mathrm{p}<0.001)$.

Conclusions: Supportive close telephone follow up significantly positively contributed to the Hp eradication success.

Keywords: Helicobacter pylori, eradication, antibiotic resistance, telephone follow up
\end{abstract}

\section{INTRODUCTION}

Helicobacter pylori's (HP) mean prevalence (carrier or asymptomatic infection) in the Northern Europe and Northern America is about 30\%. The percentage is higher than $70 \%$ in low-income nations $(1,2)$. In the past years, initial treatment for HP infection included dual antibiotic therapy with a proton-pump inhibitor. Triple eradication treatment with two antibiotics from clarithromycin, amoxicillin or metronidazole and a proton-pump inhibitor was used more commonly in the past. However, antibiotic resistant development, which reduced treatment success years during the recent years, significantly complicates the treatment (3-9). Besides, there is limited information on HP antibiotic resistance rates to guide the treatment. The selected treatment regimen should take into account local antibiotic resistance patterns (if known), previous exposure to specific antigens and allergy, cost, side effects and convenience of treatment. Lately, poor compliance seems to confound treatment in patients treated for HP eradication. Poor compliance and bacterial resistance are two important factors that lead to unacceptable HP eradication rates $(\leq 80 \%)(10,11)$.

Given this resistance setting, patient compliance to current treatment is at least as important as resistance. The present study aims to evaluate the effect of close follow-up by telephone calls on Hp eradication rates.

\section{MATERIALS AND METHOD}

This study was approved by the Ethics Committee of Sultan Abdülhamid Han Training and Research Hospital, under 
number 1491-10-16/1539. All procedures were performed adhered to the ethical rules and the Helsinki Declaration of Principles.

Patients who presented for dyspeptic complaints to the internal disease and gastroenterology clinic of our hospital between January 2017 and December 2017 were evaluated. Patients with active malignancies, hepatitis B, C, D, E with HIV and other viralinfections or autoimmune disorders were excluded from the study. Esophagogastroduodenoscopy was performed to all patients to evaluate dyspeptic symptoms where indicated. Presence of HP infection was confirmed by histopathological analysis. All biopsy samples were stained with hematoxylin and eosin. Sections with hematoxylin and eosin stains were evaluated by experienced pathologists for presence of HP using Sydney classification.

All patients included in the study were given quadruple eradication therapy consisting of tetracycline $500 \mathrm{mg} \mathrm{4x1}$, metronidazole $500 \mathrm{mg} \mathrm{3 \times 1}$, bismuth subsalicylate 262 $\mathrm{mg} 2 \times 2$ and pantoprazole $40 \mathrm{mg} 1 \times 1$. Each patient was described in detail the treatment protocol they will use before they were given their prescriptions. The patients were then divided into two equal groups of 121 individuals in each. The first group (TFG) was called every 3 days during the 14 days they received treatment and the patients were asked about treatment compliance difficulties and drug side effects. If the patients desired, they were given information on short- and long-term consequences of HP infection and they were encouraged to continue treatment. Approximately 4 weeks after treatment end, all patients were invited to return for a follow-up visit. Patients who did not return for the follow-up visit were called back again once a month for 2 consecutive months and were reminded to return for follow-up. The patients in the second group were explained the treatment protocol in detail at treatment initiation and were instructed to return for a follow-up visit 4 weeks after treatment end. They were not given support via telephone calls. Then the difference between eradication rates of the two groups was evaluated by fecal HP antigen test to assess the response to 14-days of treatment. Fresh feces samples in sterile containers were sent immediately to the laboratory. The test was repeated under optimal conditions for patients who provided diarrheic or inadequate sample. Analysis of fecal samples to assay HP antigen was performed using Hp Ag fecal enzyme-linked immunosorbent assay (ELISA) kits (ACON, San Diego, USA).

\section{Statistical Analysis}

Statistical Package for Social Sciences, version 15.0 (SPSS Inc., Chicago, IL, USA) was used for analysis. $\mathrm{p}<0.05$ was considered as statistically significant. Distributions of variables were determined by visual and analytic tests as Kolmogorov-Smirnov test. Student T and Mann Whitney U tests were used for comparisons of independent continuous variables. Dependent T test and Wilcoxon test were used for comparisons of related continuous variables. Mc Nemar test were used for comparisons of related categorical variables. Categorical independent variables were analyzed using the chi-square test or Fisher's exact test.

\section{RESULTS}

The patients were divided into two groups similar in terms for demographics, comorbidities, socioeconomic and educational status, smoking, drinking and medication history. The 242 patients' age range was $19-82$ and their mean age was $45.01 \pm 14.6$ years. Of the group, $52.1 \%(n=126)$ were women and $47.9 \%(\mathrm{n}=116)$ were men.

A total of 11 (4.5\%) individuals, 6 from the first and 5 from the second group, did not return for the first outpatient clinic follow-up after endoscopy. Four (1.7\%) individuals, two from each group, were then lost to follow-up after start of treatment. Thus, 15 (6.2\%) patients discontinued, and the study was initiated with the remaining 227 patients. Fourteen (5.8\%) individuals discontinued treatment due to side effects. Of the remaining 213 patients, 108 were randomized to TFG and 105 to NTFG (Figure 1).

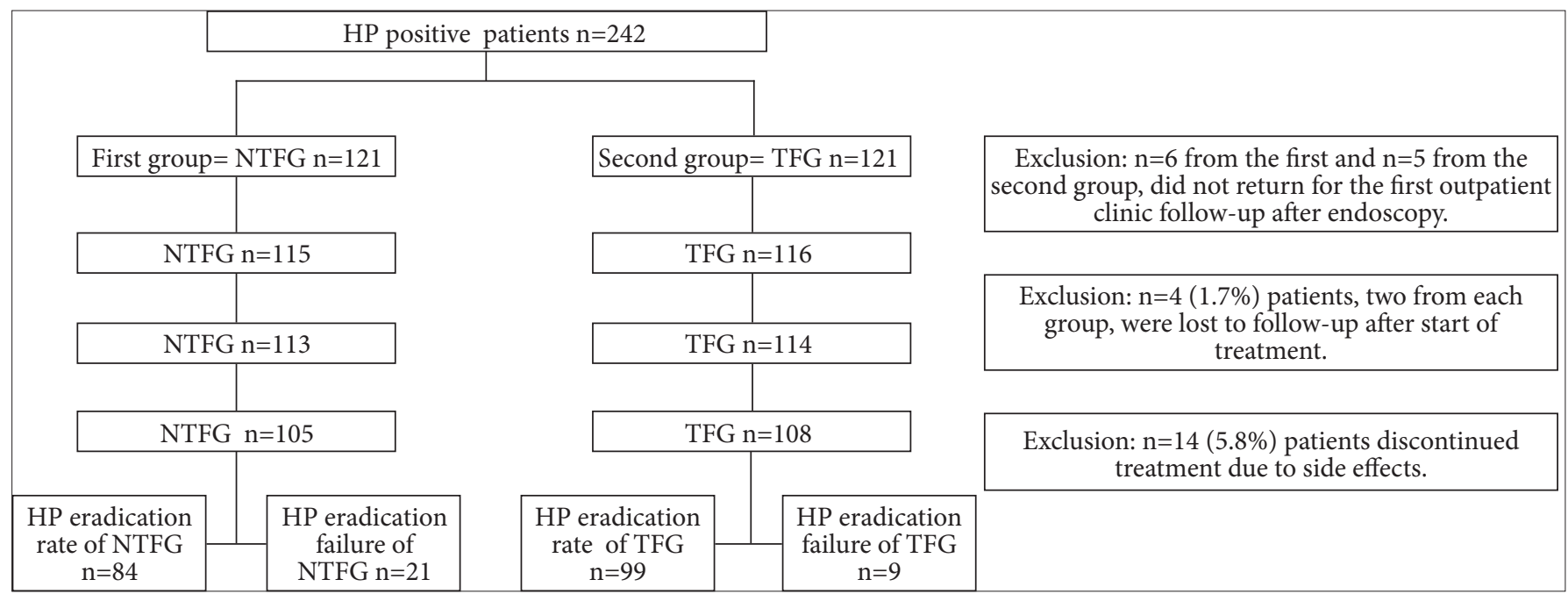

Figure 1. Study flow chart. We enrolled $242 \mathrm{HP}$ positive patients to this study and randomized to two groups 
In NTFG, eradication was achieved in $80 \%(\mathrm{n}=84)$ and could not be achieved in $20 \%(\mathrm{n}=21)$. In TGF, eradication was achieved in $91.6 \%(\mathrm{n}=99)$ and could not be achieved in $8.4 \%(n=9)(p=0.014)$ (Table, Figure 2).

\begin{tabular}{|c|c|c|c|c|}
\hline & $\begin{array}{l}\text { HP eradicated } \\
\text { (stool test } \\
\text { negative) }\end{array}$ & $\begin{array}{c}\text { HP not } \\
\text { eradicated (stool } \\
\text { test positive) }\end{array}$ & Total & $\begin{array}{c}p \\
\text { value }\end{array}$ \\
\hline NTFG & 84 & 21 & 105 & 0.014 \\
\hline TFG & 99 & 9 & 108 & \\
\hline Total & 183 & 30 & 213 & \\
\hline
\end{tabular}

NTFG: non telephone follow-up group TFG: telephone follow up group

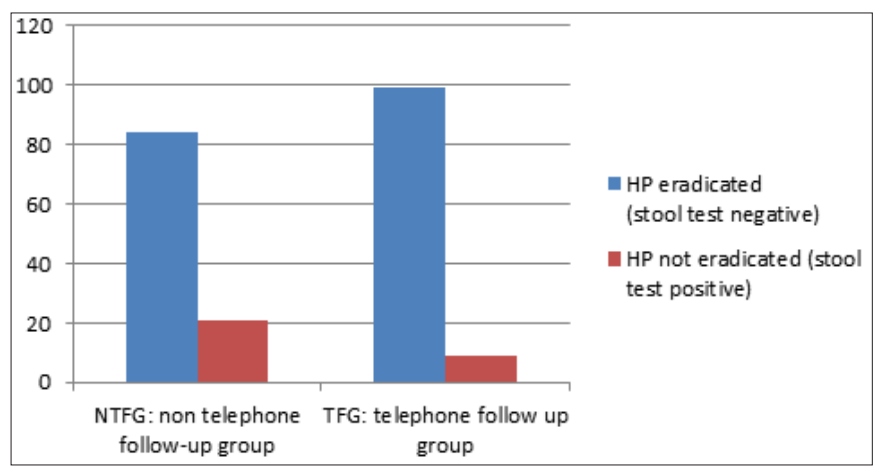

Figure 2. Graphic presentation of HP eradication rates of two study groups

\section{DISCUSSION}

To the best of our knowledge, it is the first study which investigates the effect of supportive telephone followup in Hp eradication success. Substantial changes have occurred in the diagnosis and treatment of many gastrointestinal diseases such as gastritis and ulcer when HP was first discovered in 1983 (12). In 1994, it was concluded that HP had a causal relationship with stomach carcinogenesis and is definitely a carcinogen for humans, following which the World Health Organization and World Health Organization International Agency for Research on Cancer (IARC/WHO) focused all their attention on this bacterium (13). The estimated risk of developing cancer with HP varies between 50 to $73 \%$ (14).

Many treatment protocols are used today for HP eradication. Success rates of up to $95 \%$ with standard triple eradication therapies were being mentioned before 2000s (12). The rates fell back to around 55\% over the course of years, especially with increasing resistance to clarithromycin (15). An inclination towards quadrupled protocols containing bismuth therefore occurred. Bismuth has antibacterial (against HP) and mucosal cytoprotective effects. Bismuth-containing therapies are known to be more effective in treatment of peptic ulcer and HP infection (16).
An antibiotic treatment cannot be claimed to result in success unless it is taken for an appropriate length of time at the right dosage. Poor compliance to HP eradication regimen inversely correlated with the chance of therapeutic success. It was reported that $12 \%$ of the patients prematurely discontinued eradication therapy due to side effects of the drugs used in treatment (17) Unfortunately, approved eradication regimens require combination of 3 or 4 different drugs at multiple daily doses. Complexity of treatment regimen and common occurrence of side effects are linked with patient compliance. Therefore, persuasiveness of the physician and informing the patient of possible effects are essential for therapeutic success (18).

A study performed in 2008 in Italy compared eradication rates and side effect profiles of triple therapy with lansoprazole, amoxicillin and clarithromycin and quadruple therapy with lansoprazole, metronidazole, bismuth and tetracycline for HP eradication, and included 50 subjects in the first group and 44 in the second. Treatment-emergent side effects occurred in $90 \%$ of the patients in the first group and in $95 \%$ in the second group with $6(13.6 \%)$ patients discontinuing treatment in the second group (19). As in this study, high side effect rates are seen with eradication therapy in many other studies as well, and hence total eradication rates are affected by the patients withdrawing from the study.

Many antibiotic combinations have been tried to increase eradication rates, reduce side effects and improve treatment comfort. A randomized controlled study evaluating two groups, 192 patients in each, treated either with metronidazole, tetracycline and omeprazole added to the new single-capsule bismuth or with omeprazole, amoxicillin and clarithromycin added to the new singlecapsule bismuth for eradication and side effects found no difference regarding these variables. Adverse effects were seen in $76 \%$ and in $70 \%$ of the patients in the first and second groups, respectively (20).

Attempts are being made using a number of methods to increase eradication rates with the given treatments. In some previous studies, probiotics were added with the aim of increasing eradication rates which produced no further benefits other than reducing side effects.

Gong et al. reported lower rates of $H$. pylori eradication with triple therapy compared with probiotic-supplemented triple therapy (hazard ratio [HR] 0.58; 95\% confidence interval $[\mathrm{CI}], 0.50-0.68 ; \mathrm{p}<0.05)$. Significant reductions in side effects including nausea, vomiting flatulence, epigastric pain, diarrhea, constipation, distorted taste and rash were observed (21).

In a randomized controlled study on probiotic and side effects published in the American Journal of Gastroenterology in 2002, 85 HP-positive patients were 
divided into 4 groups and started antibiotic treatment with rabeprazole $20 \mathrm{mg}$ b.i.d, clarithromycin $500 \mathrm{mg}$ b.i.d., and tinidazole $500 \mathrm{mg}$ b.i.d. The first two groups were given different probiotic replacements, the third group was given combined prebiotic replacement and the third group was given placebo, and the groups were compared for eradication rates and side effects. Tolerability was significantly better in treated patients compared with the placebo group. No difference was observed in side effect incidence across probiotic groups and HP eradication rate was almost the same between probiotic and placebo groups (22). As seen in these studies, adding a probiotic to eradication therapy has no effect on eradication rates.

Two studies were performed in China in 2015 and 2017 which evaluated eradication rates using a telephone-based close follow-up. Both studies demonstrated that followup by telephone calls had no significant effect on patients' compliance, satisfaction or HP eradication but resulted in reduced undesirable effects $(23,24)$.

Despite these similarly performed studies, we determined in our study that close follow-up by telephone calls affects eradication rates. We believe that this may be due to intercultural differences between countries and the fact that supportive information was given with each call. This demonstrated that supporting patients during treatment increases response rates to this challenging treatment although high eradication rates were seen in both groups.

Fecal antigen testing performed with a monoclonal antibody-based ELISA assay has high sensitivity and specificity but it may not always provide satisfactory results for diagnosis (25). This was the most important limitation for our study. Repeat endoscopy to evaluate eradication following treatment is not considered ethical or cost-effective. However, since eradication assessment was performed with the same method for both groups included in the study, we believe this had no impact on the study outcome.

\section{CONCLUSION}

Besides detailed informative talking about Hp treatment at the beginning, supportive treatment by telephone calls may favorably positive contributed significantly to the success of eradication.

\section{ETHICAL DECLARATIONS}

Ethics Committee Approval: This study was approved by the Ethics Committee of Sultan Abdülhamid Han Training and Research Hospital, under number 1491-10-16/1539.

Informed Consent: Written informed consent was obtained from all participants who participated in this study.
Referee Evaluation Process: Externally peer-reviewed. Conflict of Interest Statement: The authors have no conflicts of interest to declare.

Financial Disclosure: The authors declared that this study has received no financial support.

Author Contributions: All of the authors declare that they have all participated in the design, execution, and analysis of the paper, and that they have approved the final version.

\section{REFERENCES}

1. Kaplan M, Tanoglu A, Duzenli T, Tozun AN. Helicobacter pylori treatment in Turkey: Current status and rational treatment options. North Clin Istanb 2019; 7: 87-94.

2. Ruiter R, Wunderink HF, Veenendaal RA, Visser LG, de Boer MGJ. Helicobacter pylori resistance in the Netherlands: a growing problem? Neth J Med 2017; 75: 394-8.

3. Dooley CP, Cohen H, Fitzgibbons PL, et al. Prevalence of Helicobacter pylori infection and histologic gastritis in asymptomatic persons. N Engl J Med 1989; 321: 1562-6.

4. Sezikli M, Sirin G, Akkan Cetinkaya Z, et al. Comparison of the efficacy of six different Helicobacter pylori eradication regimens: greater than or equal to another. Biomedical Research 2018; 29: 1143-8.

5. Malfertheiner P, Megraud F, O’Morain CA, et al. Management of Helicobacter pylori infection-the Maastricht IV/Florence Consensus Report. Gut 2012; 61: 646-64.

6. Graham DY, Fischbach L. Helicobacter pylori treatment in the era of increasing antibiotic resistance. Gut 2010; 59: 1143-53.

7. Safavi M, Sabourian R, Foroumadi A. Treatment of Helicobacter pylori infection: current and future insights. World J Clin Cases 2016; 4: 5-19.

8. Kekilli M, Onal IK, Ocal S, et al. Inefficacy of triple therapy and comparison of two different bismuth-containing quadruple regimens as a firstline treatment option for Helicobacter pylori. Saudi J Gastroenterol 2016; 22: 366-9.

9. Pérez-Arellano E, Rodriguez-Garcia MI, Galera Rodenas AB, de la Morena-Madrigal E. Eradication of Helicobacter pylori infection with a new bismuth-based quadruple therapy in clinical practice. Gastroenterol Hepatol 2018; 41: 145-52.

10. Wermeille J, Cunningham M, Dederding JP, et al. Failure of Helicobacter pylori eradication: is poor compliance the main cause? Gastroenterol Clin Biol 2002; 26: 216-9.

11. Gisbert JP, Calvet X. Review article: the effectiveness of standard triple therapy for Helicobacter pylori has not changed over the last decade, but it is not good enough. Aliment Pharmacol Ther 2011; 34: 1255-68.

12. Ching CK, Chan YK, Ng WC. The combination of omeprazole, amoxycillin, and clarithromycin eradicates Helicobacter pylori in $95 \%$ of patients-7 days of therapy is as good as 10 days. Hong Kong Med J 1998; 4: 7-10.

13. IARC Working Group on the evaluation of carcinogenic risks to humans schistosomes, liver flukes and Helicobacter pylori . Lyon, 7-14 June 1994. IARC Monogr Eval Carcinog Risks Hum 1994; 61: 1-241.

14. Correa P, Fontham ET, Bravo JC, et al. Chemoprevention of gastric dysplasia: randomized trial of antioxidant supplements and anti-Helicobacter pylori therapy. J Natl Cancer Inst 2000; 92: $1881-8$. 
15. Wong WM, Gu Q, Wang WH, et al. Effects of primary metronidazole and clarithromycin resistance to Helicobacter pylori on omeprazole, metronidazole, and clarithromycin tripletherapy regimen in a region with high rates of metronidazole resistance. Clin Infect Dis 2003; 37: 882-9.

16. Aydın A, Günşar F, Yılmaz M, et al. Ranitidine bismuth citrate based dual and triple therapies in Helicobacter pylori eradication. Turk J Gastroenterol 1999; 10: 202-6.

17. Graham DY, Fischbach L. Helicobacter pylori treatment in the era of increasing antibiotic resistance. Gut 2010; 59: 1143-53.

18. De Francesco V, Ierardi E, Hassan C, Zullo A. Helicobacter pylori therapy: Present and future. World J Gastrointest Pharmacol Ther 2012; 3: 68-73.

19. Ching SS, Sabanathan S, Jenkinson LR. Treatment of Helicobacter pylori in surgical practice: A randomised trial of triple versus quadruple therapy in a rural district general hospital. World J Gastroenterol 2008; 14: 3855-60.

20. Xie Y, Pan X, Li Y, et al. New single capsule of bismuth, metronidazole and tetracycline given with omeprazole versus quadruple therapy consisting of bismuth, omeprazole, amoxicillin and clarithromycin for eradication of Helicobacter pylori in duodenal ulcer patients: a Chinese prospective, randomized, multicentre trial. J Antimicrob Chemother 2018 ; 73: 1681-7.

21. Gong Y, Li Y, Sun Q. Probiotics improve efficacy and tolerability of triple therapy to eradicate Helicobacter pylori: a meta-analysis of randomized controlled trials. Int J Clin Exp Med 2015; 8: 6530-43.

22. Cremonini F, Di Caro S, Covino M, et al. Effect of different probiotic preparations on anti-Helicobacter pylori therapy-related side effects: a parallel group, triple blind, placebo-controlled study. Am J Gastroenterol 2002; 97: 2744-9.

23. Wang CH, Liao ST, Yang J, et al. Effects of daily telephone-based re-education before taking medicine on Helicobacter pylori eradication: A prospective single-center study from China. World J Gastroenterol 2015; 21: 11179-84.

24. Peng X, Song L, Chen W, Zheng Y. Effect of telephone followup on compliance and Helicobacter pylori eradication in patients with Helicobacter pylori infection. Zhong Nan Da Xue Xue Bao Yi Xue Ban 2017; 42: 308-312.

25. Gisbert JP, de la Morena F, Abraira V. Accuracy of monoclonal stool antigen test for the diagnosis of $H$. pylori infection: a systematic review and meta-analysis. Am J Gastroenterol 2006; 101: 1921-30. 